\title{
NOVEL DEFECT-CORRECTION HIGH-ORDER, IN SPACE AND TIME, ACCURATE SCHEMES FOR PARABOLIC SINGULARLY PERTURBED CONVECTION-DIFFUSION PROBLEMS ${ }^{1}$
}

\author{
PIETER W. HEMKER
}

$C W I$

P.O. Box 94079, 1090 GB Amsterdam, The Netherlands

\section{GRIGORII I. SHISHKIN AND LIDIA P. SHISHKINA}

Institute of Mathematics and Mechanics, Ural Branch of the Russian Academy of Sciences 16 S. Kovalevskaya Str., 620219 Ekaterinburg, Russia

Dedicated to John J.H.Miller on the occasion of his 65th birthday.

\begin{abstract}
New high-order accurate finite difference schemes based on defect correction are considered for an initial boundary-value problem on an interval for singularly perturbed parabolic PDEs with convection; the highest space derivative in the equation is multiplied by the perturbation parameter $\varepsilon, \varepsilon \in(0,1]$. Solutions of the well-known classical numerical schemes for such problems do not converge $\varepsilon$-uniformly (the errors of such schemes depend on the value of the parameter $\varepsilon$ and are comparable with the solution itself for small values of $\varepsilon$ ). The convergence order of the existing $\varepsilon$-uniformly convergent schemes does not exceed 1 in space and time. In this paper, using a defect correction technique, we construct a special difference scheme that converges $\varepsilon$-uniformly with the second (up to a logarithmic factor) order of accuracy with respect to $x$ and with the second order of accuracy and higher with respect to t. The conditions are given which ensure the $\varepsilon$-uniform convergence of the defect-correction schemes with a rate of $\mathcal{O}\left(N^{-k} \ln ^{k} N+K^{-k_{0}}\right), k=1,2, k_{0}=1,2,3$, where $N+1$ and $K+1$ denote the number of the mesh points in $x$ and $t$, respectively. Theoretical results and the efficiency of the newly constructed schemes are confirmed by numerical experiments.
\end{abstract}

2000 Mathematics Subject Classification: 65M06; 65M12; 65M15.

Keywords: singular perturbation problem, convection-diffusion equations, $\varepsilon$-uniform fitted mesh method, defect correction, higher-order accuracy.

\section{Introduction}

In this paper we consider the initial boundary-value problem on an interval for singularly perturbed parabolic PDEs with convection. The highest space derivative in the equation is

\footnotetext{
1 This research was supported in part by the Dutch Research Organisation NWO (grant No. 047.008.007), by the Russian Foundation for Basic Research (grant No. 01-01-01022) and by the Enterprise Ireland Research Grant SC-2000-070.
} 
multiplied by an arbitrarily small positive parameter $\varepsilon ; \varepsilon \in(0,1]$. When the perturbation parameter $\varepsilon$ tends to zero, the solution of such a problem typically exhibits a boundary layer in the outflow boundary region. This gives rise to difficulties when classical discretization methods are applied, because the errors in the numerical solution depend appreciably on the value of the parameter $\varepsilon$, namely, the errors of standard methods can be large when the step-size in $x$ becomes comparable with $\varepsilon$. Thus, in connection with such behavior of the errors for standard numerical methods applied to the problem in question, it is of interest to develop special numerical methods whose errors would be independent of the parameter $\varepsilon$ and, would depend only on the number of mesh points, i.e., $\varepsilon$-uniformly convergent methods. Such methods have been suggested in the literature for a number of singularly perturbed elliptic and parabolic equations (see, for example, [1-3,10,11,13,14] and also the bibliography therein). It should be noted that the rate of $\varepsilon$-uniform convergence of the known special schemes for parabolic convection-diffusion equations is $\mathcal{O}\left(N^{-1} \ln N+K^{-1}\right)$, i.e., it is of order no more than one, where $N$ and $K$ define the numbers of the nodes in the meshes with respect to $x$ and $t$. However, the well-known classical difference methods of high-order accuracy with respect to $x$ and/or $t$ for the same problems (see, for example, $[9,12]$ and also the bibliography therein), generally speaking, do not converge $\varepsilon$-uniformly. Thus, it is necessary to construct $\varepsilon$-uniformly convergent schemes of high-order accuracy with respect to $x$ and/or $t$ for a class of singularly perturbed convection-diffusion problems. Besides, a higher order accuracy in time can considerably reduce computational expenses.

Defect correction techniques proved to be efficient for constructing $\varepsilon$-uniformly convergent schemes of high-order accuracy with respect to $t$ in the case of singularly perturbed reaction-diffusion and convection-diffusion problems (see, for example, [4-6]. Therefore, this technique seems attractive because of its possible use in constructing high-order accurate schemes in $x$ and $t$ for singularly perturbed problems under consideration.

In the present paper, $\varepsilon$-uniformly convergent schemes of high-order accuracy in time and space are constructed, also based on the defect correction principle, in the case of boundary value problems for singularly perturbed parabolic convection-diffusion equations. The efficiency of the newly constructed schemes is confirmed by numerical experiments.

\section{The studied class of initial boundary-value problems}

On the domain $G=D \times(0, T], D=(0,1)$ with the boundary $S=\bar{G} \backslash G$, we consider the following singularly perturbed parabolic equation subject to the Dirichlet boundary conditions $^{2}$ :

$$
\begin{aligned}
L_{(2.1)} u(x, t) \equiv & \left\{\varepsilon a(x, t) \frac{\partial^{2}}{\partial x^{2}}+b(x, t) \frac{\partial}{\partial x}-c(x, t)-p(x, t) \frac{\partial}{\partial t}\right\} u(x, t)=f(x, t), \\
& (x, t) \in G, \\
u(x, t)= & \varphi(x, t), \quad(x, t) \in S .
\end{aligned}
$$

For $S=S_{0} \cup S^{L}$, we distinguish the lateral boundary $S^{L}=\{(x, t): x=0$ or $x=1$, $0<t \leqslant T\}$ and the initial boundary $S_{0}=\{(x, t): 0 \leqslant x \leqslant 1, t=0\}$. In (2.1) $a(x, t)$, $b(x, t), c(x, t), p(x, t), f(x, t),(x, t) \in \bar{G}$ and $\varphi(x, t),(x, t) \in S$ are sufficiently smooth and bounded functions which satisfy

$$
0<a_{0} \leqslant a(x, t), \quad 0<b_{0} \leqslant b(x, t), \quad 0<p_{0} \leqslant p(x, t), \quad c(x, t) \geqslant 0, \quad(x, t) \in \bar{G} .
$$

\footnotetext{
${ }^{2}$ The notation is such that the operator $L_{(a . b)}$ is first introduced in equation $(a . b)$.
} 
Here $a_{0}, b_{0}, p_{0}$ are positive constants independent of $\varepsilon$. The perturbation parameter $\varepsilon$ in (2.1a) may take any values from the half-open unit interval

$$
\varepsilon \in(0,1]
$$

When the parameter $\varepsilon$ tends to zero, the solution exhibits a layer in the neighborhood of the set $S_{1}^{L}=\{(x, t): x=0,0 \leqslant t \leqslant T\}$, i.e., the left-hand side of the lateral boundary. This layer is described by an ordinary differential equation (an ordinary boundary layer).

\section{Difference scheme on an arbitrary mesh}

To solve problem (2.1) we first consider a classical finite difference method, that is a base scheme. On the set $\bar{G}$ we introduce the rectangular mesh

$$
\bar{G}_{h}=\bar{\omega} \times \bar{\omega}_{0},
$$

where $\bar{\omega}$ is the (possibly) non-uniform mesh of nodal points, $x^{i}$, in $[0,1], \bar{\omega}_{0}$ is a uniform mesh on the interval $[0, T] ; N$ and $K$ are the numbers of intervals in the meshes $\bar{\omega}$ and $\bar{\omega}_{0}$, respectively. We define $\tau=T / K, h^{i}=x^{i+1}-x^{i}, h=\max _{i} h^{i}, h \leqslant M / N, G_{h}=G \cap \bar{G}_{h}$, $S_{h}=S \cap \bar{G}_{h} \cdot{ }^{3}$

For problem (2.1) we use the difference scheme [12]

$$
\begin{aligned}
\Lambda_{(3.2)} z(x, t) & =f(x, t), & & (x, t) \in G_{h}, \\
z(x, t) & =\varphi(x), & & (x, t) \in S_{h} .
\end{aligned}
$$

Here

$$
\begin{aligned}
\Lambda_{(3.2)} z(x, t) & \equiv\left\{\varepsilon a(x, t) \delta_{\bar{x} \widehat{x}}+b(x, t) \delta_{x}-c(x, t)-p(x, t) \delta_{\bar{t}}\right\} z(x, t), \quad(x, t) \in G_{h}, \\
\delta_{\bar{x} \widehat{x}} z\left(x^{i}, t\right) & =2\left(h^{i-1}+h^{i}\right)^{-1}\left(\delta_{x} z\left(x^{i}, t\right)-\delta_{\bar{x}} z\left(x^{i}, t\right)\right), \\
\delta_{\bar{x}} z\left(x^{i}, t\right) & =\left(h^{i-1}\right)^{-1}\left(z\left(x^{i}, t\right)-z\left(x^{i-1}, t\right)\right), \\
\delta_{x} z\left(x^{i}, t\right) & =\left(h^{i}\right)^{-1}\left(z\left(x^{i+1}, t\right)-z\left(x^{i}, t\right)\right), \\
\delta_{\bar{t}} z\left(x^{i}, t\right) & =\tau^{-1}\left(z\left(x^{i}, t\right)-z\left(x^{i}, t-\tau\right)\right),
\end{aligned}
$$

$\delta_{x} z(x, t)$ and $\delta_{\bar{x}} z(x, t), \delta_{\bar{t}} z(x, t)$ are the forward and backward differences, and the difference operator $\delta_{\bar{x} \widehat{x}} z(x, t)$ is an approximation of the operator $\frac{\partial^{2}}{\partial x^{2}} u(x, t)$ on the non-uniform mesh.

The difference scheme (3.2), (3.1) is monotone. By means of the maximum principle and taking into account a priori estimates of the derivatives (see Theorem A.1 in the Appendix A) we find that the solution of the difference scheme (3.2), (3.1) converges for a fixed value of the parameter $\varepsilon$ :

$$
|u(x, t)-z(x, t)| \leqslant M\left(\varepsilon^{-2} N^{-1}+\tau\right), \quad(x, t) \in \bar{G}_{h}
$$

This error bound for the classical difference scheme is clearly not $\varepsilon$-uniform.

The proof of (3.3) follows the lines of the classical convergence proof for monotone difference schemes (see $[12,14]$ ). This results in the following theorem.

\footnotetext{
${ }^{3}$ Here and in what follows $M$ (or $m$ ) denote generic sufficiently large (small) positive constants (possibly subscripted) that do not depend on $\varepsilon$ and on the discretization parameters.
} 
Theorem 3.1. Assume in equation (2.1) that a, b, c, p, $f \in H^{(\vartheta+2 n-2)}(\bar{G}), \varphi \in$ $H^{(\vartheta+2 n)}\left(S_{0}\right) \cap H^{(\vartheta+2 n)}\left(\bar{S}^{L}\right), \vartheta>4, n=0$, and let the compatibility conditions (A.1) with $n=0$ (see Appendix A) be satisfied. Then, for a fixed value of the parameter $\varepsilon$, the solution of (3.2), (3.1) converges to the solution of (2.1) with an error bound given by (3.3).

\section{The $\varepsilon$-uniformly convergent scheme}

Here we discuss an $\varepsilon$-uniformly convergent fitted mesh method for (2.1) by taking a special mesh condensed in the neighborhood of the boundary layer. The location of the nodes is determined properly from the a priori estimates of the solution and its derivatives. The way to construct the mesh for problem $(2.1)$ is the same as in $[4,5,15]$. More specifically, we take

$$
\bar{G}_{h}=\bar{G}_{h}^{*}=\bar{\omega}^{*}(\sigma) \times \bar{\omega}_{0},
$$

where $\bar{\omega}_{0}$ is the uniform mesh with the step-size $\tau=T / K$, i.e., $\bar{\omega}_{0}=\bar{\omega}_{0(3.1)}$, and $\bar{\omega}^{*}=\bar{\omega}^{*}(\sigma)$ is a special piecewise uniform mesh depending on the parameter $\sigma \in \mathbb{R}$, which depends in turn on $\varepsilon$ and $N$. We take

$$
\sigma=\sigma_{(4.1)}(\varepsilon, N)=\min \left\{1 / 2, m^{-1} \varepsilon \ln N\right\},
$$

where $m$ is an arbitrary number from the interval $\left(0, m_{0}\right), m_{0}=\min _{\bar{G}}\left[(a(x, t))^{-1} b(x, t)\right]$. The mesh $\bar{\omega}^{*}(\sigma)$ is constructed as follows. The interval $[0,1]$ is divided into two parts $[0, \sigma]$, $[\sigma, 1], \sigma \leqslant 1 / 2$, and in each part we use a uniform mesh, with $N / 2$ subintervals in $[0, \sigma]$ and $[\sigma, 1]$.

Theorem 4.1. Let the hypotheses of Theorem 3.1 be fulfilled. Then the solution of (3.2), (4.1) converges $\varepsilon$-uniformly to the solution of problem (2.1) and the following error estimate holds:

$$
|u(x, t)-z(x, t)| \leqslant M\left(N^{-1} \ln N+\tau\right), \quad(x, t) \in \bar{G}_{h}^{*} .
$$

The proof of this theorem can be found in $[14,16]$.

\section{Improved accuracy}

\subsection{High-order accurate schemes based on defect correction}

In this section we construct a new numerical method based on defect correction, which also converges $\varepsilon$-uniformly to the solution of the boundary-value problem, but with an order of accuracy higher than in (4.2).

The technique used in this paper to improve accuracy is similar to that from [4-6]. For the difference scheme (3.2), (4.1) the error in the approximation of the partial derivative $(\partial / \partial t) u(x, t)$ is caused by the divided difference $\delta_{\bar{t}} z(x, t)$ and is associated with the truncation error given by

$$
\frac{\partial u}{\partial t}(x, t)-\delta_{\bar{t}} u(x, t)=2^{-1} \tau \frac{\partial^{2} u}{\partial t^{2}}(x, t)-6^{-1} \tau^{2} \frac{\partial^{3} u}{\partial t^{3}}(x, t-\theta), \quad \theta \in[0, \tau] .
$$

Therefore, for the approximation of $(\partial / \partial t) u(x, t)$ we now use the expression

$$
\delta_{\bar{t}} u(x, t)+\tau \delta_{\bar{t} \bar{t}} u(x, t) / 2 \text {, where } \delta_{\bar{t} \bar{t}} u(x, t) \equiv \delta_{t \bar{t}} u(x, t-\tau) .
$$


Note that $\delta_{t \bar{t}} u(x, t)$ is the second central divided difference. In a similar way, the truncation error for the forward difference $\delta_{x} z(x, t)$ is defined by the formula

$$
\frac{\partial u}{\partial x}(x, t)-\delta_{x} u(x, t)=-2^{-1} h^{i} \frac{\partial^{2}}{\partial x^{2}} u\left(x+\theta_{1}, t\right), \quad \theta_{1} \in\left[0, h^{i}\right], \quad x=x^{i}, \quad h^{i}=x^{i+1}-x^{i} .
$$

For the approximation of $(\partial / \partial x) u(x, t)$ we use the relation $\delta_{x} u(x, t)-h^{i} \delta_{\bar{x} \widehat{x}} u(x, t) / 2$. We can evaluate a better approximation than $(3.2 \mathrm{a})$ by defect correction:

$$
\Lambda_{(3.2)} z^{c}(x, t)=f(x, t)+2^{-1} \tau p(x, t) \frac{\partial^{2} u}{\partial t^{2}}(x, t)+2^{-1} h^{i} b(x, t) \frac{\partial^{2} u}{\partial x^{2}}(x, t), \quad x=x^{i},
$$

with $x \in \bar{\omega}$ and $t \in \bar{\omega}_{0}$, where $\bar{\omega}$ and $\bar{\omega}_{0}$ are as in (3.1); $\tau$ is the step-size of the mesh $\bar{\omega}_{0}$ and $h^{i}=x^{i+1}-x^{i}$ is the local step-size of the mesh $\bar{\omega}_{0} ; z^{c}(x, t)$ is the "corrected" solution. Instead of $\left(\partial^{2} / \partial t^{2}\right) u(x, t)$ and $\left(\partial^{2} / \partial x^{2}\right) u(x, t)$ we shall use $\delta_{\bar{t} \bar{t}} z(x, t)$ and $\delta_{\bar{x} \widehat{x}} z(x, t)$, respectively, where $z(x, t),(x, t) \in G_{h(4.1)}$ is the solution of the difference scheme (3.2), (4.1). We may expect that the new solution $z^{c}(x, t)$ has an $\varepsilon$-uniform consistency error of order $\mathcal{O}\left(\tau^{2}\right)$ with respect to the variable $t$. This is true, as will be shown in Section 5.2 in Theorem 5.1. Concerning the variable $x$, the consistency error of the corrected solution on uniform meshes is $\mathcal{O}\left(N^{-2}\right)$ for a fixed $\varepsilon$. However, in the case of special piecewise uniform meshes the order of $\varepsilon$-uniform convergence with respect to $x$ is expected as $\mathcal{O}\left(N^{-2} \ln ^{2} N\right)$, that is, the second order up to a logarithmic factor (see Section 5.5).

Moreover, in a similar way we can construct a difference approximation with a convergence order higher than two with respect to the time variable and $\mathcal{O}\left(N^{-2} \ln ^{2} N\right)$ with respect to the space variable $\varepsilon$-uniformly.

For notational convenience, on the mesh $\bar{G}_{h}$ we write the finite difference scheme (3.2) in the form

$$
\begin{array}{rlrl}
\Lambda_{(3.2)} z^{(1)}(x, t) & =f(x, t), & & (x, t) \in G_{h}, \\
z^{(1)}(x, t) & =\varphi(x, t), & (x, t) \in S_{h},
\end{array}
$$

where $z^{(1)}(x, t)$ is the uncorrected solution. This scheme is referred to as the base scheme.

\subsection{The defect correction scheme of second-order accuracy in time}

We denote by $\delta_{k \bar{t}} z(x, t)$ the backward difference of order $k$ :

$$
\begin{aligned}
& \delta_{k \bar{t}} z(x, t)=\left(\delta_{k-1 \bar{t}} z(x, t)-\delta_{k-1 \bar{t}} z(x, t-\tau)\right) / \tau, \quad t \geqslant k \tau, \quad k \geqslant 1 ; \\
& \delta_{0 \bar{t}} z(x, t)=z(x, t), \quad(x, t) \in \bar{G}_{h} .
\end{aligned}
$$

To construct the difference schemes of second-order accuracy in $\tau$ in (5.1), instead of $\left(\partial^{2} / \partial t^{2}\right) u(x, t)$ we use $\delta_{2 \bar{t}} z(x, t)$, the second divided difference of the solution to the discrete problem $(3.2),(4.1)$. For the corrected solution $z^{(2)}(x, t)$ we solve the problem for $(x, t) \in G_{h}$

$$
\begin{aligned}
\Lambda_{(3.2)} z^{(2)}(x, t) & =f(x, t)+\psi^{(1)}(x, t), & & (x, t) \\
z^{(2)}(x, t) & =\varphi(x, t), & & (x, t) \in G_{h},
\end{aligned}
$$

Here

$$
\psi^{(1)}(x, t) \equiv\left\{\begin{array}{ll}
p(x, t) 2^{-1} \tau \frac{\partial^{2}}{\partial t^{2}} u(x, 0), & t=\tau \\
p(x, t) 2^{-1} \tau \delta_{2 \bar{t}} z^{(1)}(x, t), & t \geqslant 2 \tau
\end{array}\right\}, \quad(x, t) \in G_{h}
$$


the derivative $\frac{\partial^{2} u}{\partial t^{2}}(x, 0)$ can be obtained from equation $(2.1 \mathrm{a})$. We shall call $z^{(2)}(x, t)$ the solution of difference scheme (5.3), (5.2), (4.1) (or shortly, (5.3), (4.1)).

For simplicity, in the remainder of this section we suppose that the coefficients $a(x, t)$, $b(x, t)$ do not depend on $t$ :

$$
a(x, t)=a(x), \quad b(x, t)=b(x), \quad(x, t) \in \bar{G},
$$

and we take a homogeneous initial condition:

$$
\varphi(x, t)=0, \quad(x, t) \in S_{0}
$$

Under conditions (5.4), (5.5), the following estimate holds for the solution of problem (5.3), (4.1):

$$
\left|u(x, t)-z^{(2)}(x, t)\right| \leqslant M\left[N^{-1} \ln N+\tau^{2}\right], \quad(x, t) \in \bar{G}_{h} .
$$

Theorem 5.1. Let conditions (5.4), (5.5) hold and assume in equation (2.1) that $a, b$, $c, p, f \in H^{(\vartheta+2 n-2)}(\bar{G}), \varphi \in H^{(\vartheta+2 n)}\left(S_{0}\right) \cap H^{(\vartheta+2 n)}\left(\bar{S}^{L}\right), \vartheta>4, n=1$, and also let the compatibility conditions (A.1) with $n=1$ (see Appendix A) be satisfied. Then for the solution of difference scheme (5.3), (4.1) the estimate (5.6) is valid.

The proof of Theorem 5.1 can be found in [6].

Remark 5.1. The conclusion of Theorem 5.1 remains also valid for a number of cases when the coefficients $a$ and $b$ depend on $x, t$, for example, when the condition $(a(x, t))^{-1} b(x, t)=g(x),(x, t) \in \bar{G}$ is fulfilled; here $a, b \in H^{(\vartheta+2 n-2)}(\bar{G})$ (see the assumption of Theorem 5.1) and condition (2.1c) must be satisfied.

This remark holds also for Theorems 5.2, 5.3, 5.4.

\subsection{The defect correction scheme of third-order accuracy in time}

The above procedure can be used to obtain an arbitrarily large order of time-accuracy. Here we only show how to construct the difference scheme of third-order accuracy in time. On the grid $\bar{G}_{h}$ we consider the difference scheme

$$
\begin{aligned}
\Lambda_{(3.2)} z^{(3)}(x, t) & =f(x, t)+\psi^{(2)}(x, t),, & & (x, t) \in G_{h}, \\
z^{(3)}(x, t) & =\varphi(x, t), & & (x, t) \in S_{h} .
\end{aligned}
$$

Here

$$
\psi^{(2)}(x, t) \equiv\left\{\begin{array}{ll}
p(x, t)\left(C_{11} \tau \frac{\partial^{2}}{\partial t^{2}} u(x, 0)+C_{12} \tau^{2} \frac{\partial^{3}}{\partial t^{3}} u(x, 0)\right), & t=\tau \\
p(x, t)\left(C_{21} \tau \frac{\partial^{2}}{\partial t^{2}} u(x, 0)+C_{22} \tau^{2} \frac{\partial^{3}}{\partial t^{3}} u(x, 0)\right), & t=2 \tau \\
p(x, t)\left(C_{31} \tau \delta_{2 \bar{t}} z^{(2)}(x, t)+C_{32} \tau^{2} \delta_{3 \bar{t}} z^{(1)}(x, t)\right), & t \geqslant 3 \tau
\end{array}\right\}, \quad(x, t) \in G_{h},
$$

$z^{(1)}(x, t)$ and $z^{(2)}(x, t)$ are the solutions of problems (5.2), (4.1) and (5.3), (4.1), respectively; the derivatives $\left(\partial^{2} / \partial t^{2}\right) u(x, 0),\left(\partial^{3} / \partial t^{3}\right) u(x, 0)$ are again obtained from equation (2.1a). The 
coefficients $C_{i j}$ are chosen to satisfy the following conditions:

$$
\begin{aligned}
\frac{\partial}{\partial t} u(x, t) & =\delta_{\bar{t}} u(x, t)+C_{11} \tau \frac{\partial^{2}}{\partial t^{2}} u(x, t-\tau)+C_{12} \tau^{2} \frac{\partial^{3}}{\partial t^{3}} u(x, t-\tau)+\mathcal{O}\left(\tau^{3}\right), \\
\frac{\partial}{\partial t} u(x, t) & =\delta_{\bar{t}} u(x, t)+C_{21} \tau \frac{\partial^{2}}{\partial t^{2}} u(x, t-2 \tau)+C_{22} \tau^{2} \frac{\partial^{3}}{\partial t^{3}} u(x, t-2 \tau)+\mathcal{O}\left(\tau^{3}\right), \\
\frac{\partial}{\partial t} u(x, t) & =\delta_{\bar{t}} u(x, t)+C_{31} \tau \delta_{2 \bar{t}} u(x, t)+C_{32} \tau^{2} \delta_{3 \bar{t}} u(x, t)+\mathcal{O}\left(\tau^{3}\right) .
\end{aligned}
$$

It follows that

$$
C_{11}=C_{21}=C_{31}=1 / 2, \quad C_{12}=C_{32}=1 / 3, \quad C_{22}=5 / 6 .
$$

By $z^{(3)}(x, t)$ we denote the solution of the difference scheme (5.7), (4.1) and again, for simplicity, we assume that the homogeneous initial condition holds

$$
\varphi(x, t)=0, \quad f(x, 0)=0, \quad(x, t) \in S_{0} .
$$

Under conditions (5.4), (5.8) the following estimate holds for the solution of difference scheme (5.7), (4.1):

$$
\left|u(x, t)-z^{(3)}(x, t)\right| \leqslant M\left[N^{-1} \ln N+\tau^{3}\right], \quad(x, t) \in \bar{G}_{h} .
$$

Theorem 5.2. Let conditions (5.4), (5.8) hold and assume in equation (2.1) that a, $b, c, p, f \in H^{(\vartheta+2 n-2)}(\bar{G}), \varphi \in H^{(\vartheta+2 n)}\left(S_{0}\right) \cap H^{(\vartheta+2 n)}\left(\bar{S}^{L}\right), \vartheta>4, n=2$, and let the compatibility conditions (A.1) with $n=2$ (see Appendix A) be satisfied. Then for the solution of scheme (5.7), (4.1) estimate (5.9) is valid.

The proof of Theorem 5.2 can be found in [6].

In a similar way we could construct difference schemes with an arbitrarily high order of accuracy in time

$$
\mathcal{O}\left(N^{-1} \ln N+\tau^{n+1}\right), \quad n>2 .
$$

\subsection{A scheme with improved convergence in space}

We now describe a defect-correction scheme which is used to improve accuracy with respect to the space variable. On mesh (3.1) we consider the discrete problem

$$
\begin{aligned}
& \Lambda_{(3.2)} z^{[2]}(x, t)=f(x, t)+\psi^{[1]}(x, t), \quad(x, t) \in G_{h}, \\
& z^{[2]}(x, t)=\varphi(x, t), \quad(x, t) \in S_{h},
\end{aligned}
$$

where

$$
\psi^{[1]}(x, t) \equiv b(x, t) 2^{-1} h^{i-1} \delta_{\bar{x} \widehat{x}} z^{(1)}(x, t), \quad x=x^{i}, \quad(x, t) \in G_{h} ;
$$

$z^{(1)}(x, t),(x, t) \in G_{h}$ is the solution of the base scheme (5.2), (3.1); $h^{i-1}=x^{i}-x^{i-1}, x^{i-1}, x^{i} \in$ $\bar{\omega}$. Note that $\delta_{x} z\left(x^{i}, t\right)-2^{-1} h^{i-1} \delta_{\bar{x} \widehat{x}} z\left(x^{i}, t\right)=\delta_{\widetilde{x}} z\left(x^{i}, t\right)$, where $\delta_{\widetilde{x}} z\left(x^{i}, t\right)$ is the first central difference derivative, $\delta_{\widetilde{x}} z\left(x^{i}, t\right)=\left(h^{i}+h^{i-1}\right)^{-1}\left(z\left(x^{i+1}, t\right)-z\left(x^{i-1}, t\right)\right)$. We call the function $z^{[2]}(x, t),(x, t) \in G_{h}$, the solution of difference scheme (5.10), (5.2), (3.1) (or, shortly, $(5.10),(3.1))$.

In contrast to mesh (4.1), in the case of scheme (5.10), (5.2) we use the special fitted mesh with a rather extended (as compared with mesh (4.1)) neighborhood of the small stepsize in $x$ : 


$$
\bar{G}_{h}^{*}=\bar{G}_{h(4.1 a)}^{*},
$$

where $\bar{\omega}^{*}=\bar{\omega}_{(4.1 a)}^{*}(\sigma)$, provided that

$$
\sigma=\sigma_{(5.11)}(\varepsilon, N)=\min \left\{1 / 2, l m^{-1} \varepsilon \ln N\right\},
$$

$m=m_{(4.1)}, \quad l \geqslant 3$ is an arbitrary number.

Assume that conditions (5.4), (5.5) are valid. Then we obtain the following $\varepsilon$-uniform estimate for the solution of problem (5.10), (5.11):

$$
\left|u(x, t)-z^{[2]}(x, t)\right| \leqslant M\left[N^{-2} \ln ^{2} N+\tau\right], \quad(x, t) \in \bar{G}_{h}^{*} .
$$

Theorem 5.3. Let the hypotheses of Theorem 5.1 be fulfilled. Then the solution of the difference scheme (5.10), (5.11) satisfies estimate (5.12).

The proof of this theorem is given in the Appendix B.

\subsection{A scheme with improved convergence in time and space}

At last we give the difference scheme of higher order accuracy with respect to $x$ and $t$.

On mesh (3.1) we approximate the boundary-value problem (2.1) by the difference scheme

$$
\begin{aligned}
\Lambda_{(3.2)} z^{[2, n+1]}(x, t) & =f(x, t)+\psi^{[1]}(x, t)+\psi^{(n)}(x, t), & & (x, t) \in G_{h}, \\
z^{[2, n+1]}(x, t) & =\varphi(x, t), & & (x, t) \in S_{h}, \quad n=1,2 .
\end{aligned}
$$

Here

$$
\psi^{[1]}(x, t)=\psi_{(5.10)}^{[1]}(x, t), \quad \psi^{(1)}(x, t)=\psi_{(5.3)}^{(1)}(x, t), \quad \psi^{(2)}(x, t)=\psi_{(5.7)}^{(2)}(x, t) .
$$

When calculating the functions $\psi^{[1]}(x, t), \psi^{(1)}(x, t)$ and $\psi^{(2)}(x, t)$ we use respectively the functions $z^{(1)}(x, t)$ and $z^{(1)}(x, t), z^{(2)}(x, t)$, where $z^{(1)}(x, t)$ and $z^{(2)}(x, t)$ are the solutions of problems (5.2), (3.1) and (5.3), (3.1) as before. We call the function $z^{[2, n+1]}(x, t),(x, t) \in$ $G_{h(3.1)}$, the solution of difference scheme (5.13), (3.1). To solve the boundary-value problem (2.1) more accurately, we use the difference scheme (5.13) on the special mesh (5.11).

In the case of conditions (5.4), (5.5) we obtain the estimate

$$
\left|u(x, t)-z^{[2,2]}(x, t)\right| \leqslant M\left[N^{-2} \ln ^{2} N+\tau^{2}\right], \quad(x, t) \in \bar{G}_{h}^{*} .
$$

In the case where conditions (5.4) and (5.8) are satisfied, we have the estimate

$$
\left|u(x, t)-z^{[2,3]}(x, t)\right| \leqslant M\left[N^{-2} \ln ^{2} N+\tau^{3}\right], \quad(x, t) \in \bar{G}_{h}^{*} .
$$

Theorem 5.4. Let the hypotheses of Theorem 5.1 (Theorem 5.2) be fulfilled. Then for the solution of the difference scheme (5.13), (5.11) estimate (5.14) (estimate (5.15)) holds.

The proof of this theorem is similar to the proof of Theorem 5.3. 


\section{Numerical results for the scheme of improved accuracy in space and time}

Let us consider some numerical results for scheme (5.13), (5.11) for $n=1$.

To see the effect of the special scheme in practice, we find the solution of the following boundary-value problem:

$$
\begin{array}{rlrl}
L_{(6.1)} u(x, t) & \equiv\left\{\varepsilon \frac{\partial^{2}}{\partial x^{2}}+\frac{\partial}{\partial x}-\frac{\partial}{\partial t}\right\} u(x, t)=f(x, t), & & (x, t) \in G, \\
u(x, t) & =\varphi(x, t), & (x, t) \in S,
\end{array}
$$

where

$$
\begin{aligned}
& u(0, t)=t^{4}+t^{5}, \quad u(1, t)=t+t^{5}, \quad 0<t \leqslant T=1 ; \quad u(x, 0)=0, \quad 0<x \leqslant 1 ; \\
& f(x, t)=12 \varepsilon t x^{2}+4 t x^{3}-x^{4}-5 t^{4} .
\end{aligned}
$$

It should be noted that the solution of this problem is singular.

It is very attractive to use the analytical solution of problem (6.1) for the computation of the errors in the approximate solution, as was done in $[4,5]$. But here a suitable (for computation) representation of the solution $u(x, t)$ is unknown. Instead of the exact solution, it is possible to use the solution of the discrete problem on a very fine mesh. But this method is not effective because the analysis of the order of accuracy for a defect-correction scheme requires a very dense mesh that leads not only to large computational expenses but also to large round-off errors.

Hence, we use the method from [7], which differs from the above-mentioned techniques. The solution of problem (6.1) is represented in the form of the sum

$$
u(x, t)=U(x, t)+V^{(1)}(x, t)+v(x, t), \quad(x, t) \in \bar{G},
$$

where $U(x, t)=t x^{4}+t^{5}, V^{(1)}(x, t)$ is the main singular part (two first terms) of the asymptotic expansion of the solution of problem $(6.1)$, and $v(x, t)$ is the remainder term, which is a sufficiently small smooth function. The function $V^{(1)}(x, t)$ has a sufficiently simple analytical representation

$$
V^{(1)}(x, t)=V_{0}(x, t)+V_{1}(x, t), \quad(x, t) \in \bar{G},
$$

where

$$
\begin{aligned}
V_{0}(x, t) & =t^{4} \Psi(x), \quad \Psi(x)=\frac{\exp \left(-\varepsilon^{-1} x\right)-\exp \left(-\varepsilon^{-1}\right)}{1-\exp \left(-\varepsilon^{-1}\right)}, \\
V_{1}(x, t) & =-4 t^{3} x \frac{\exp \left(-\varepsilon^{-1} x\right)}{1-\exp \left(-\varepsilon^{-1}\right)}, \\
\left|V_{0}(x, t)\right| & \leqslant M, \quad\left|V_{1}(x, t)\right| \leqslant M \varepsilon, \quad(x, t) \in \bar{G} .
\end{aligned}
$$

The function $v(x, t)$ is the solution of the problem

$$
\begin{gathered}
L_{(6.1)} v(x, t)=f_{0}(x, t), \quad(x, t) \in G, \\
v(0, t)=0, \quad v(1, t)=-V_{1}(1, t), \quad 0<t \leqslant T, \quad v(x, 0)=0, \quad 0<x<1,
\end{gathered}
$$

with 


$$
f_{0}(x, t)=-4 t^{2} \frac{t \exp (-1 / \varepsilon)+3 x \exp (-x / \varepsilon)}{1-\exp (-1 / \varepsilon)} .
$$

For the function $v(x, t)$ the following estimate holds:

$$
\left|\frac{\partial^{k+k_{0}}}{\partial x^{k} \partial t^{k_{0}}} v(x, t)\right| \leqslant M \varepsilon^{2}\left[1+\varepsilon^{-k}\right], \quad(x, t) \in \bar{G}, \quad k+2 k_{0} \leqslant 4, \quad k \leqslant 3 .
$$

Then the function $v(x, t)$ and the product $\varepsilon^{2}\left(\partial^{4} / \partial x^{4}\right) v(x, t)$ are $\varepsilon$-uniformly bounded. Thus, we can consider $v(x, t)$ as the regular part of this solution and, moreover, $v(x, t)$ is of order $\mathcal{O}\left(\varepsilon^{2}\right)$, according to (6.4).

(1.) For the chosen value of $\varepsilon$, we solve the discrete problem, which approximates the boundary-value problem (6.3), on the finest available mesh $\bar{G}_{h}=\bar{G}_{h(5.11)}^{*}$ for $N=K=2048$, and there are no difficulties in finding the function $v(x, t)=v_{\varepsilon}^{2048}(x, t)$ and the reference solution $u_{(6.2)}(x, t)$ which can be practically taken as the exact solution

$$
u_{(6.2)}(x, t)=u_{\varepsilon}^{2048}(x, t)=U(x, t)+V^{(1)}(x, t)+v_{\varepsilon}^{2048}(x, t) .
$$

(2.) Further, for solving problem (6.1), we use successively scheme (5.2), (5.11) and the defect correction scheme (5.13), (5.11) to find the functions $z^{(1)}(x, t)$ and $z^{[2,2]}(x, t)$, respectively. Note that $z^{(1)}(x, t)$ is the uncorrected solution, $z^{[2,2]}(x, t)$ is the corrected solution. Then we compute the maximum pointwise errors $E_{z^{j}}(N, K, \varepsilon)$ from the formula

$$
E_{z^{j}}(N, K, \varepsilon)=\max _{(x, t) \in \bar{G}_{h}}\left|z^{j}(x, t)-u^{*}(x, t)\right|, \quad j=(1),[2,2] .
$$

Here $u^{*}(x, t)$ is the linear interpolation obtained from the reference solution $u_{\varepsilon}^{2048}(x, t)$ corresponding to the numerical solution $z^{j}(x, t), j=(1),[2,2]$ for $N=K=2^{i}, i=2,3, \ldots, 10$.

The computational process (1.) and (2.) is repeated for all the values of $\varepsilon=2^{-n}$, $n=0,1,2, \ldots, 13$. As a result, we get $E_{z^{(1)}}(N=K, \varepsilon)$ and $E_{z^{[2,2]}}(N=K, \varepsilon)$ for the functions $z^{(1)}(x, t)$ and $z^{[2,2]}(x, t)$, respectively, for various values of $\varepsilon, N=K$ (see Table 1 ).

Analyzing these results from Table 1 , we see $\varepsilon$-uniform convergence of the numerical solutions with increasing $N=K$ for both of the functions $z^{(1)}(x, t)$ and $z^{[2,2]}(x, t)$. Further we observe that the errors for the function $z^{[2,2]}(x, t)$ are smaller than the errors for the function $z^{(1)}(x, t)$, that is, the new defect correction scheme gives the better result.

If we calculate the ratios of the maximum pointwise errors

$$
R_{z^{j}}(N)=\frac{\bar{E}_{z^{j}}\left(2^{-1} N\right)}{\bar{E}_{z^{j}}(N)}, \quad j=(1),[2,2]
$$

for the functions $z^{(1)}(x, t)$ and $z^{[2,2]}(x, t)$ and place them in Table 2, then we can see that the order of convergence is almost one for the function $z^{(1)}(x, t)$ and almost two for the function $z^{[2,2]}(x, t)$, which corresponds to the theoretical results. 
Table 1. Errors for the model problem (6.1), namely, $\boldsymbol{E}_{\boldsymbol{z}^{(1)}}(\boldsymbol{N}=\boldsymbol{K}, \boldsymbol{\varepsilon})$ for the special scheme (5.2), (4.1) and $\boldsymbol{E}_{\boldsymbol{z}^{[2,2]}}(\boldsymbol{N}=\boldsymbol{K}, \boldsymbol{\varepsilon})$ with improved convergence in time and space for the defect correction scheme $(5.13),(5.11)$

\begin{tabular}{|c|c|c|c|c|c|c|c|c|c|}
\hline$\varepsilon \backslash N$ & 4 & 8 & 16 & 32 & 64 & 128 & 256 & 512 & 1024 \\
\hline & \multicolumn{9}{|c|}{$z^{(1)}$} \\
\hline $2^{-0}$ & $3.00-1$ & $1.61-1$ & $8.41-2$ & $4.28-2$ & $2.15-2$ & $1.07-2$ & $5.31-3$ & $2.58-3$ & $1.21-3$ \\
\hline $2^{-1}$ & $4.46-1$ & $2.46-1$ & $1.29-1$ & $6.59-2$ & $3.32-2$ & $1.67-2$ & $8.33-3$ & $4.14-3$ & $2.04-3$ \\
\hline $2^{-2}$ & 5.69-1 & $3.29-1$ & $1.72-1$ & $8.77-2$ & $4.43-2$ & $2.22-2$ & $1.12-2$ & $5.59-3$ & $2.81-3$ \\
\hline $2^{-3}$ & 7.07-1 & $3.92-1$ & $2.06-1$ & $1.06-1$ & $5.40-2$ & $2.73-2$ & $1.37-2$ & $6.89-3$ & $3.47-3$ \\
\hline $2^{-4}$ & $8.08-1$ & $4.34-1$ & $2.45-1$ & $1.33-1$ & $6.96-2$ & $3.57-2$ & $1.81-2$ & $9.15-3$ & $4.61-3$ \\
\hline $2^{-5}$ & $8.46-1$ & $4.68-1$ & 2.66-1 & $1.54-1$ & $8.88-2$ & $5.08-2$ & $2.78-2$ & $1.42-2$ & $7.18-3$ \\
\hline $2^{-6}$ & $8.54-1$ & $4.80-1$ & $2.77-1$ & $1.60-1$ & $9.30-2$ & 5.33-2 & $2.99-2$ & $1.65-2$ & $9.01-3$ \\
\hline $2^{-7}$ & $8.56-1$ & $4.86-1$ & $2.82-1$ & $1.63-1$ & $9.57-2$ & 5.49-2 & $3.08-2$ & $1.70-2$ & $9.30-3$ \\
\hline $2^{-8}$ & $8.57-1$ & 4.88-1 & $2.85-1$ & $1.65-1$ & $9.71-2$ & 5.57-2 & $3.13-2$ & $1.73-2$ & $9.44-3$ \\
\hline $2^{-9}$ & $8.57-1$ & $4.90-1$ & $2.86-1$ & $1.67-1$ & $9.77-2$ & 5.61-2 & $3.15-2$ & $1.74-2$ & $9.51-3$ \\
\hline $2^{-10}$ & $8.57-1$ & $4.90-1$ & $2.87-1$ & $1.67-1$ & $9.81-2$ & 5.63-2 & $3.16-2$ & $1.75-2$ & $9.55-3$ \\
\hline $2^{-11}$ & $8.57-1$ & 4.91-1 & $2.88-1$ & $1.67-1$ & $9.82-2$ & 5.64-2 & $3.17-2$ & $1.75-2$ & $9.57-3$ \\
\hline $2^{-12}$ & $8.57-1$ & $4.91-1$ & $2.88-1$ & $1.67-1$ & $9.83-2$ & 5.64-2 & $3.17-2$ & $1.75-2$ & $9.58-3$ \\
\hline $2^{-13}$ & $8.57-1$ & $4.91-1$ & $2.88-1$ & $1.68-1$ & $9.84-2$ & 5.64-2 & $3.17-2$ & $1.75-2$ & $9.58-3$ \\
\hline \multirow[t]{2}{*}{$\overline{\mathbf{E}}_{\mathbf{z}^{(\mathbf{1})}}(\mathbf{N})$} & 8.57-1 & 4.91-1 & 2.88-1 & 1.68-1 & 9.84-2 & 5.64-2 & 3.17-2 & $1.75-2$ & 9.58-3 \\
\hline & \multicolumn{9}{|c|}{$z^{[2,2]}$} \\
\hline $2^{-0}$ & $1.29-1$ & $3.81-2$ & $1.04-2$ & $2.59-3$ & $5.49-4$ & $7.80-5$ & $1.37-4$ & $1.61-4$ & $1.68-4$ \\
\hline $2^{-1}$ & $1.89-1$ & 5.73-2 & $1.54-2$ & $3.97-3$ & $9.74-4$ & $2.19-4$ & $5.88-5$ & $7.79-5$ & $8.42-5$ \\
\hline $2^{-2}$ & 2.51-1 & 7.31-2 & $1.95-2$ & $5.03-3$ & $1.30-3$ & $3.58-4$ & $1.25-4$ & $6.77-5$ & $5.38-5$ \\
\hline $2^{-3}$ & $3.01-1$ & $8.83-2$ & $2.43-2$ & $6.58-3$ & $1.76-3$ & $4.95-4$ & $1.70-4$ & $8.75-5$ & $6.67-5$ \\
\hline $2^{-4}$ & $3.63-1$ & $1.05-1$ & $3.51-2$ & $1.06-2$ & $3.14-3$ & 8.99-4 & $2.67-4$ & $9.99-5$ & $5.76-5$ \\
\hline $2^{-5}$ & $3.96-1$ & $1.28-1$ & $4.14-2$ & $1.51-2$ & $5.27-3$ & $1.89-3$ & $6.29-4$ & $1.85-4$ & $6.56-5$ \\
\hline $2^{-6}$ & $4.34-1$ & $1.52-1$ & $4.65-2$ & $1.63-2$ & $5.80-3$ & $2.02-3$ & $6.74-4$ & $2.13-4$ & $7.44-5$ \\
\hline $2^{-7}$ & $4.52-1$ & $1.64-1$ & $5.24-2$ & $1.68-2$ & $6.06-3$ & $2.10-3$ & $6.97-4$ & $2.15-4$ & $7.05-5$ \\
\hline $2^{-8}$ & $4.60-1$ & $1.70-1$ & 5.54-2 & $1.71-2$ & $6.18-3$ & $2.14-3$ & 7.09-4 & $2.18-4$ & $6.99-5$ \\
\hline $2^{-9}$ & 4.64-1 & $1.73-1$ & $5.69-2$ & $1.72-2$ & $6.24-3$ & $2.16-3$ & 7.14-4 & $2.19-4$ & $6.99-5$ \\
\hline $2^{-10}$ & $4.66-1$ & $1.75-1$ & $5.76-2$ & $1.73-2$ & $6.27-3$ & $2.17-3$ & $7.17-4$ & $2.20-4$ & $7.00-5$ \\
\hline $2^{-11}$ & $4.67-1$ & $1.76-1$ & $5.80-2$ & $1.73-2$ & $6.28-3$ & $2.17-3$ & $7.18-4$ & $2.20-4$ & $7.01-5$ \\
\hline $2^{-12}$ & 4.68-1 & $1.76-1$ & $5.82-2$ & $1.74-2$ & $6.28-3$ & $2.17-3$ & 7.19-4 & $2.20-4$ & $7.01-5$ \\
\hline $2^{-13}$ & $4.68-1$ & $1.76-1$ & 5.83-2 & $1.74-2$ & 6.29-3 & $2.17-3$ & 7.19-4 & $2.20-4$ & 7.01-5 \\
\hline$\overline{\mathbf{E}}_{\mathbf{z}}{ }^{[2,2]}(\mathbf{N})$ & 4.68-1 & $1.76-1$ & 5.83-2 & 1.74-2 & 6.29-3 & 2.17-3 & 7.19-4 & 2.20-4 & 7.01-5 \\
\hline
\end{tabular}

In this table the function $\boldsymbol{E}_{\boldsymbol{z}^{j}}(\boldsymbol{N}=\boldsymbol{K}, \boldsymbol{\varepsilon})$ is defined by (6.5). In the bottom line $\overline{\boldsymbol{E}}_{\boldsymbol{z}^{j}}(\boldsymbol{N})$ gives the computed maximum pointwise errors for each column, i.e., $\overline{\boldsymbol{E}}_{\boldsymbol{z}^{j}}(\boldsymbol{N})=$ $\max _{\varepsilon} E_{z^{j}}(N=K, \varepsilon)$; here $j=(1),[2,2]$.

Table 2. Ratios of the maximum pointwise errors for the functions $\boldsymbol{z}^{(\mathbf{1})}$ and $\boldsymbol{z}^{[\mathbf{2 , 2}]}$

\begin{tabular}{|c|cccccccc|}
\hline $\mathrm{N}$ & 8 & 16 & 32 & 64 & 128 & 256 & 512 & 1024 \\
\hline $\mathbf{R}_{\mathrm{z}^{(1)}}(\mathrm{N})$ & 1.74 & 1.70 & 1.71 & 1.71 & 1.74 & 1.78 & 1.81 & 1.83 \\
\hline $\mathbf{R}_{\mathrm{z}^{[2,2]}}(\mathrm{N})$ & 2.66 & 3.02 & 3.35 & 2.77 & 2.90 & 3.02 & 3.23 & 3.14 \\
\hline
\end{tabular}




\section{Conclusions}

In this paper we have shown theoretically that the use of a defect correction technique for solving the class of boundary value problems for a singularly perturbed parabolic convectiondiffusion equation allows us to construct effectively $\varepsilon$-uniformly convergent schemes with the second (up to a logarithmic factor) order of accuracy with respect to $x$ and with the second, third, and higher orders of accuracy with respect to $t$.

The numerical example is given where it is shown that the order of convergence with respect to the space variable is $\mathcal{O}\left(N^{-2} \ln ^{2} N\right)$, which corresponds to the theoretical results.

\section{Appendix A. A priori estimates of the solution and its derivatives}

In this section we rely on the a priori estimates for the solution of problem (2.1) on the domain $G=D \times[0, T]$ and its derivatives as derived for elliptic and parabolic equations in $[14,15,17]$.

We denote by $H^{(\vartheta)}(\bar{G})=H^{\vartheta, \vartheta / 2}(\bar{G})$ the Hölder space, where $\vartheta$ is an arbitrary positive number [8]. We suppose that the functions $f(x, t)$ and $\varphi(x, t)$ satisfy compatibility conditions at the corner points, so that the solution of the boundary-value problem is smooth for each fixed value of the parameter $\varepsilon$.

For simplicity, we assume that the following conditions hold at the corner points $S_{0} \cap \bar{S}^{L}$ :

$$
\begin{aligned}
\frac{\partial^{k}}{\partial x^{k}} \varphi(x, t) & =0, \quad \frac{\partial^{k_{0}}}{\partial t^{k_{0}}} \varphi(x, t)=0, & & k+2 k_{0} \leqslant[\vartheta]+2 n, \\
\frac{\partial^{k+k_{0}}}{\partial x^{k} \partial t^{k_{0}}} f(x, t) & =0, & & k+2 k_{0} \leqslant[\vartheta]+2 n-2,
\end{aligned}
$$

where $[\vartheta]$ is the integer part of the number $\vartheta, \vartheta>0, n \geqslant 0$ is an integer. We also suppose that $[\vartheta]+2 n \geqslant 2$.

Using interior a priori estimates and estimates up to the boundary for the regular function $\widetilde{u}(\xi, t)$ (see $[8]$ ), where $\widetilde{u}(\xi, t)=u(x(\xi), t), \xi=x / \varepsilon$, we find the estimate for $(x, t) \in \bar{G}$

$$
\left|\frac{\partial^{k+k_{0}}}{\partial x^{k} \partial t^{k_{0}}} u(x, t)\right| \leqslant M \varepsilon^{-k}, \quad k+2 k_{0} \leqslant 2 n+4, \quad n \geqslant 0 .
$$

This estimate holds, for example, for

$$
u \in H^{(2 n+4+\nu)}(\bar{G}), \quad \nu>0,
$$

where $\nu$ is some small number.

For example, (A.3) is guaranteed for the solution of (2.1) if the coefficients satisfy inclusions $a, c, p, f \in H^{(\vartheta+2 n-2)}(\bar{G}), \varphi \in H^{(\vartheta+2 n)}\left(S_{0}\right) \cap H^{(\vartheta+2 n)}\left(\bar{S}^{L}\right), \vartheta>4, n \geqslant 0$ and condition (A.1) is fulfilled.

In fact, we need a more accurate estimate than (A.2). Therefore, we represent the solution of the boundary-value problem (2.1) in the form of the sum

$$
u(x, t)=U(x, t)+W(x, t), \quad(x, t) \in \bar{G},
$$

where $U(x, t)$ represents the regular part, and $W(x, t)$ the singular part, i.e., the boundary layer. The function $U(x, t)$ is the smooth solution of equation (2.1a) satisfying condition 
(2.1b) for $t=0$ and $x=1$. For example, under suitable assumptions for the data of the problem, we can consider the solution of the boundary-value problem for equation (2.1a) smoothly continued onto the domain $\bar{G}^{*}$ extended beyond $S_{1}^{L}\left(\bar{G}^{*}\right.$ is a sufficiently large neighborhood $\bar{G}$ beyond $S_{1}^{L}$ ). On the domain $\bar{G}$ the coefficients and the initial value of the extended problem are the same as for (2.1). Then the function $U(x, t)$ is the restriction (on $\bar{G})$ of the solution to the extended problem, and $U \in H^{(2 n+4+\nu)}(\bar{G}), \nu>0$. The function $W(x, t)$ is the solution of a boundary-value problem for the parabolic equation

$$
\begin{aligned}
L_{(2.1)} W(x, t) & =0, & & (x, t) \in G, \\
W(x, t) & =u(x, t)-U(x, t), & & (x, t) \in S .
\end{aligned}
$$

If (A.3) is true, then $W \in H^{(2 n+4+\nu)}(\bar{G})$. Now, for the functions $U(x, t)$ and $W(x, t)$ we derive the estimates

$$
\begin{aligned}
\left|\frac{\partial^{k+k_{0}}}{\partial x^{k} \partial t^{k_{0}}} U(x, t)\right| & \leqslant M, \\
\left|\frac{\partial^{k+k_{0}}}{\partial x^{k} \partial t^{k_{0}}} W(x, t)\right| & \leqslant M \varepsilon^{-k} \exp \left(-m_{(A .7)} \varepsilon^{-1} x\right), \\
(x, t) & \in \bar{G}, \quad k+2 k_{0} \leqslant 2 n+2,
\end{aligned}
$$

where $m_{(A .7)}$ is a constant from the interval $\left(0, m_{0}\right), m_{0}=\min _{\bar{G}}\left[(a(x, t))^{-1} b(x, t)\right]$. Estimates (A.6) and (A.7) hold, for example, when

$$
U, W \in H^{(2 n+4+\nu)}(\bar{G}), \quad \nu>0 .
$$

Inclusions (A.8) are guaranteed if $a, c, p, f \in H^{(\vartheta+2 n-2)}(\bar{G}), \varphi \in H^{(\vartheta+2 n)}\left(S_{0}\right) \cap$ $H^{(\vartheta+2 n)}\left(\bar{S}^{L}\right), \vartheta>4, n \geqslant 0$ and condition (A.1) is fulfilled. We summarize these results in the following theorem.

Theorem A.1. Assume in equation (2.1) that a, b, c, p, $f \in H^{(\vartheta+2 n-2)}(\bar{G}), \varphi \in$ $H^{(\vartheta+2 n)}\left(S_{0}\right) \cap H^{(\vartheta+2 n)}\left(\bar{S}^{L}\right), \vartheta>4, n \geqslant 0$ and let condition (A.1) be fulfilled. Then, for the solution $u(x, t)$ of problem (2.1) and for its components from the representation (A.4), it follows that $u, U, W \in H^{(\vartheta+2 n)}(\bar{G})$ and that estimates (A.2), (A.6), (A.7) hold.

See the proof of the theorem in [14].

\section{Appendix B. The proof of Theorem 5.3}

In order to see the idea of the proof of Theorem 5.3, we make some constructions in the case of a model problem.

On the set

$$
\bar{D}=[0,1]
$$

with the boundary $\Gamma=\bar{D} \backslash D$, we consider the following boundary-value problem:

$$
\begin{aligned}
L u(x) & \equiv\left\{\varepsilon a(x) \frac{d^{2}}{d x^{2}}+b(x) \frac{d}{d x}-c(x)\right\} u(x)=f(x), \quad x \in D, \\
u(x) & =\varphi(x), \quad x \in \Gamma .
\end{aligned}
$$


The functions $a(x), b(x), c(x), f(x)$ are sufficiently smooth and bounded functions which satisfy

$$
a(x) \geqslant a_{0}>0, \quad b(x) \geqslant b_{0}>0, \quad c(x) \geqslant 0, \quad x \in \bar{D} .
$$

On the fitted mesh

$$
\bar{D}_{h}=\bar{D}_{h}^{*}
$$

where

$$
\bar{D}_{h}^{*}=\bar{\omega}_{(4.1 a)}^{*}(\sigma), \quad \sigma=\sigma(\varepsilon, N)=\min \left\{1 / 2, l m^{-1} \varepsilon \ln N\right\},
$$

$m$ is a constant from the interval $\left(0, m_{0}\right), m_{0}=\min _{\bar{D}}\left[(a(x))^{-1} b(x)\right], l \geqslant 3$, we use the following difference scheme for problem (B.2), (B.1):

$$
\begin{aligned}
\Lambda z(x) & \equiv\left\{\varepsilon a(x) \delta_{\bar{x} \widehat{x}}+b(x) \delta_{x}-c(x)\right\} z(x)=f(x), \quad x \in D_{h}, \\
z(x) & =\varphi(x), \quad x \in \Gamma_{h} .
\end{aligned}
$$

Here $D_{h}=D \cap \bar{D}_{h}, \Gamma_{h}=\Gamma \cap \bar{D}_{h}$.

We decompose the solution of problem (B.2), (B.1) into the sum of its regular and singular components

$$
u(x)=U(x)+V(x), \quad x \in \bar{D},
$$

which are constructed similarly to the components from (A.4). In an analogous way, the solution of problem (B.4), (B.3) can be decomposed into the sum

$$
z(x)=z_{U}(x)+z_{V}(x), \quad x \in \bar{D}_{h} .
$$

Here, in the case of a sufficiently smooth function $v(x), x \in \bar{D}$, we denote by $z_{v}(x), x \in \bar{D}_{h}$ the solution of the problem

$$
\Lambda z(x)=L v(x), \quad x \in D_{h}, \quad z(x)=v(x), \quad x \in \Gamma_{h} .
$$

The function $z^{c}(x), x \in \bar{D}_{h}$, i.e., the solution of the defect-correction scheme

$$
\begin{aligned}
\Lambda_{(B .4)} z^{c}(x) & =f(x)+b(x) 2^{-1} h^{i-1} \delta_{\bar{x} \widehat{x}} z(x), & & x \in D_{h}, \\
z^{c}(x) & =\varphi(x), & & x \in \Gamma_{h},
\end{aligned}
$$

is decomposed analogously

$$
z^{c}(x)=z_{U}^{c}(x)+z_{V}^{c}(x), \quad x \in \bar{D}_{h},
$$

where the components $z_{U}^{c}(x)$ and $z_{V}^{c}(x)$ are the solutions of the defect-correction schemes corresponding to the components of the decomposition (B.5a). Assume that

$$
\omega_{v}(x)=z_{v}(x)-v(x), \quad \omega_{v}^{c}(x)=z_{v}^{c}(x)-v(x), \quad x \in \bar{D}_{h},
$$

where $v(x)$ is one of the functions $(U(x)$ or $V(x))$ from the representation (B.5a).

Let us estimate the component $\omega_{V}^{c}(x)$. Note that the functions $\omega_{V}^{c}(x), \omega_{V}(x), z_{V}^{c}(x)$, $x \in \bar{D}_{h}$ are the solutions of the following problems:

$$
\begin{aligned}
\Lambda \omega_{V}^{c}(x) & =\left(L-\Lambda^{*}\right) V(x)+2^{-1} h^{i-1} b(x) \delta_{\bar{x} \widehat{x}} \omega_{V}(x), \quad x \in D_{h}, \\
\omega_{V}^{c}(x) & =0, \quad x \in \Gamma_{h} ; \\
\Lambda \omega_{V}(x) & =(L-\Lambda) V(x), \quad x \in D_{h}, \\
\omega_{V}(x) & =0, \quad x \in \Gamma_{h} ; \\
\Lambda z_{V}^{c}(x) & =2^{-1} h^{i-1} b(x)\left\{\delta_{\bar{x} \widehat{x}} V(x)+\delta_{\bar{x} \widehat{x}} \omega_{V}(x)\right\}, \quad x \in D_{h}, \\
z_{V}^{c}(x) & =V(x), \quad x \in \Gamma_{h},
\end{aligned}
$$


where

$$
\Lambda^{*} z(x) \equiv\left\{\varepsilon a(x) \delta_{\widehat{x} \widehat{x}}+b(x) \delta_{\widetilde{x}}-c(x)\right\} z(x),
$$

$\delta_{\widetilde{x}} z(x)$ is the centered difference, $\delta_{\widetilde{x}} z\left(x^{i}\right)=\left(h^{i}+h^{i-1}\right)^{-1}\left(z\left(x^{i+1}\right)-z\left(x^{i-1}\right)\right)$.

Using the majorant function technique, we find

$$
\begin{aligned}
\left|\omega_{V}(x)\right| & \leqslant M\left\{N^{-1} \min \left[\ln N, \varepsilon^{-1}\right]+N^{-l}\right\}, \quad x \in \bar{D}_{h} ; \\
\left|h^{i-1} \delta_{\bar{x} \widehat{x}} \omega_{V}(x)\right| & \leqslant M N^{-l+1}, \quad x \in D_{h}, \quad x \geqslant \sigma ; \quad x=x^{i} .
\end{aligned}
$$

Further, considering problem (B.7), (B.3) on the set $x \leqslant \sigma$, we get the estimate for $\left|h^{i-1} \delta_{\bar{x} \widehat{x}} \omega_{V}(x)\right|$ on $x \in D_{h}$ for $x<\sigma$ :

$$
\begin{gathered}
\left|h^{i-1} \delta_{\bar{x} \widehat{x}} \omega_{V}(x)\right| \leqslant M\left\{N^{-2} \min ^{2}\left[\ln N, \varepsilon^{-1}\right] \varepsilon^{-1} \exp \left(-m \varepsilon^{-1} x\right)+N^{-l+1}\right\}, \\
x \in D_{h}, \quad x<\sigma, \quad m=m_{(5.2)} .
\end{gathered}
$$

Considering problem (B.8) and taking into account estimates (B.9b), (B.10) and also the estimate for $h^{i-1} \delta_{\bar{x} \widehat{x}} V(x)$, we find the estimates

$$
\begin{aligned}
& \left|z_{V}^{c}(x)\right| \leqslant M\left[\exp \left(-m \varepsilon^{-1} x\right)+N^{-l+1}\right], \quad x \in \bar{D}_{h} ; \\
& \left|\omega_{V}^{c}(x)\right| \leqslant M N^{-l+1}, \quad x \in \bar{D}_{h}, \quad x \geqslant \sigma .
\end{aligned}
$$

On the set $x \in \bar{D}_{h}, x \leqslant \sigma$, the solution of problem (B.6) satisfies the estimate

$$
\left|\omega_{V}^{c}(x)\right| \leqslant M\left\{N^{-2} \min ^{2}\left[\ln N, \varepsilon^{-1}\right]+N^{-l+1}\right\}, \quad x \in \bar{D}_{h}, \quad x \leqslant \sigma .
$$

Thus, by virtue of estimates (B.11b), (B.12), we obtain

$$
\left|V(x)-z_{V}^{c}(x)\right| \leqslant M N^{-2} \min ^{2}\left[\ln N, \varepsilon^{-1}\right], \quad x \in \bar{D}_{h} .
$$

To estimate the component $\omega_{U}^{c}(x)$ we use solutions of problems which are similar to problems (B.6)-(B.8)

$$
\begin{aligned}
\Lambda \omega_{U}^{c}(x) & =\left(L-\Lambda^{*}\right) U(x)+2^{-1} h^{i-1} b(x) \delta_{\bar{x} \widehat{x}} \omega_{U}(x), \quad x \in D_{h}, \\
\omega_{U}^{c}(x) & =0, \quad x \in \Gamma_{h} ; \\
\Lambda \omega_{U}(x) & =(L-\Lambda) U(x), \quad x \in D_{h}, \\
\omega_{U}(x) & =0, \quad x \in \Gamma_{h} ; \\
\Lambda z_{U}^{c}(x) & =f(x)+2^{-1} h^{i-1} b(x)\left\{\delta_{\bar{x} \widehat{x}} U(x)+\delta_{\bar{x} \widehat{x}} \omega_{U}(x)\right\}, \quad x \in D_{h}, \\
z_{U}^{c}(x) & =U(x), \quad x \in \Gamma_{h} .
\end{aligned}
$$

In this case we obtain the estimate

$$
\left|U(x)-z_{U}^{c}(x)\right| \leqslant M N^{-2} \min \left[\ln N, \varepsilon^{-1}\right], \quad x \in \bar{D}_{h} .
$$

Thus, by virtue of (B.13), (B.14), we have

$$
|u(x)-z(x)| \leqslant M N^{-2} \min ^{2}\left[\ln N, \varepsilon^{-1}\right], \quad x \in \bar{D}_{h} .
$$

The analysis of convergence of schemes (5.10), (5.11) in the case of problem (2.1) is similar to that for scheme (B.4), (B.3) for problem (B.2). The technique of investigating 
approximations to derivatives with respect to $x$ and $t$ with using difference derivatives of discrete solutions is given in [6], where defect-correction schemes of higher-order accuracy with respect to the time variable are considered.

We represent the functions $z^{(1)}(x, t), z^{[2]}(x, t),(x, t) \in \bar{G}_{h}$, i.e., the components of the solution to problem (5.10), (5.2), (5.11), as a sum of the functions corresponding to the decomposition (A.4)

$$
\begin{aligned}
z^{[2]}(x, t) & =z_{U}^{[2]}(x, t)+z_{V}^{[2]}(x, t), \\
z^{(1)}(x, t) & =z_{U}^{(1)}(x, t)+z_{V}^{(1)}(x, t), \quad(x, t) \in \bar{G}_{h} ;
\end{aligned}
$$

assume that

$$
\begin{aligned}
\omega_{v}^{(1)}(x, t) & =z_{v}^{(1)}(x, t)-v(x, t), \\
\omega_{v}^{[2]}(x, t) & =z_{v}^{[2]}(x, t)-v(x, t), \quad(x, t) \in \bar{G}_{h},
\end{aligned}
$$

where $v(x, t)$ is any function in representation (A.4). The functions $\omega_{v}^{[2]}(x, t), \omega_{v}^{(1)}(x, t)$, $z_{v}^{[2]}(x, t)$ are the solutions of the problems

$$
\begin{aligned}
\Lambda \omega_{v}^{[2]}(x, t) & =\left(L-\Lambda^{*}\right) v(x, t)+2^{-1} h^{i-1} b(x, t) \delta_{\widehat{x} \widehat{x}} \omega_{v}^{(1)}(x, t), \\
\Lambda \omega_{v}^{(1)}(x, t) & =(L-\Lambda) v(x, t), \quad(x, t) \in G_{h}, \\
\omega_{v}^{[2]}(x, t) & =\omega_{v}^{(1)}(x, t)=0, \quad(x, t) \in S_{h} ; \\
\Lambda z_{v}^{[2]}(x, t) & =f_{v}(x, t)+2^{-1} h^{i-1} b(x, t)\left\{\delta_{\widehat{x} \widehat{x}} v(x, t)+\delta_{\bar{x} \widehat{x}} \omega_{v}^{(1)}(x, t)\right\}, \quad(x, t) \in G_{h}, \\
z_{v}^{[2]}(x, t) & =v(x, t), \quad(x, t) \in S_{h},
\end{aligned}
$$

where $f_{v}(x, t)=0$ for $v(x, t)=V(x, t), f_{v}(x, t)=f(x, t)$ for $v(x, t)=U(x, t)$.

Taking into account a priori estimates (A.6), (A.7) for the components from (A.4), we find

$$
\begin{aligned}
& \left|\delta_{t}^{k} \omega_{V}^{(1)}(x, t)\right| \leqslant \begin{cases}M\left[\left(N^{-1} \min \left[\ln N, \varepsilon^{-1}\right]+\varepsilon N_{0}^{-1}\right) \exp \left(-m \varepsilon^{-1} x\right)+N^{-l}\right], & x<\sigma ; \\
M N^{-l} q^{n}, & x \geqslant \sigma ;\end{cases} \\
& (x, t) \in \bar{G}_{h}^{k} \text {; } \\
& \left|h^{i-1} \delta_{\bar{x} \widehat{x}} \omega^{(1)}(x, t)\right| \leqslant\left\{\begin{aligned}
& M\left[\left(N^{-2} \min ^{2}\left[\ln N, \varepsilon^{-1}\right]+\varepsilon N^{-1} N_{0}^{-1}\right) \varepsilon^{-1} \exp \left(-m \varepsilon^{-1} x\right)+N^{-l+1}\right], \\
& x<\sigma ; \\
& M N^{-l+1}, x \geqslant \sigma ; \quad(x, t) \in G_{h} ; \quad k=0,1,2,
\end{aligned}\right.
\end{aligned}
$$

where

$$
\begin{aligned}
\delta_{t}^{k} z(x, t) & =\delta_{t}\left(\delta_{t}^{k-1} z(x, t)\right), \quad k \geqslant 1, \quad \delta_{t}^{1} z(x, t)=\delta_{t} z(x, t), \quad \delta_{t}^{0} z(x, t)=z(x, t) ; \\
\bar{G}_{h}^{k} & =\bar{G}_{h} \cap\left\{t \leqslant T-k h_{t}\right\}, \quad m=m_{(A .7)}, \quad q=\left(1+m_{1} \varepsilon^{-1} N^{-1}\right), \\
n & =\left(h^{(2)}\right)^{-1}(x-\sigma), \quad h^{(2)}=h_{(5.11)}^{(2)}, \quad \sigma=\sigma_{(5.11)} .
\end{aligned}
$$

Taking into account the above estimates, we obtain

$$
\left|\omega_{V}^{[2]}(x, t)\right| \leqslant M\left[N^{-2} \min ^{2}\left[\ln N, \varepsilon^{-1}\right]+N_{0}^{-2}\right], \quad(x, t) \in \bar{G}_{h} .
$$


Further we find the estimates for the difference derivatives of the function $\omega_{U}^{(1)}(x, t)$

$$
\begin{aligned}
&\left|\delta_{t}^{k} \omega_{U}^{(1)}(x, t)\right| \leqslant M\left[N^{-1}+N_{0}^{-1}\right], \quad(x, t) \in \bar{G}_{h}^{k}, \quad k=0,1,2 ; \\
&\left|\delta_{x} \omega_{U}^{(1)}(x, t)\right| \leqslant \begin{cases}M\left[\varepsilon^{-1}\left(N^{-1}+N_{0}^{-1}\right) \exp \left(-m \varepsilon^{-1} x\right)+N^{-1}+N_{0}^{-1}\right], & x<\sigma ; \\
M\left[N^{-1}+N_{0}^{-1}\right], & x \geqslant \sigma ;\end{cases} \\
&(x, t) \in \bar{G}_{h} \backslash S_{2} .
\end{aligned}
$$

Taking these estimates into account, we establish the estimate

$$
\left|h^{i-1} \delta_{\bar{x} \widehat{x}} \omega_{U}^{(1)}(x, t)\right| \leqslant\left\{\begin{array}{l}
M\left\{N^{-2} \min ^{2}\left[\ln N, \varepsilon^{-1}\right]+N_{0}^{-2}\right\}\left[\varepsilon^{-1} \exp \left(-m \varepsilon^{-1} x\right)+1\right], \\
M \varepsilon^{-1}\left[N^{-2}+N_{0}^{-2}\right], \quad x>\sigma ; \quad(x, t) \in G_{h} .
\end{array}\right.
$$

Making estimate (B.18a) for $x>\sigma$ more precise, we obtain

$$
\begin{gathered}
\left|h^{i-1} \delta_{\bar{x} \widehat{x}} \omega_{U}^{(1)}(x, t)\right| \leqslant M\left[N^{-2}+N_{0}^{-2}+\left(N^{-2}+N_{0}^{-2}\right)\left(\varepsilon+N^{-1}\right)^{-1} q^{n-1}\right], \\
(x, t) \in G_{h}, \quad x>\sigma .
\end{gathered}
$$

Taking estimates (B.18) into consideration, we find the estimate

$$
\left|\omega_{U}^{[2]}(x, t)\right| \leqslant M\left\{N^{-2} \min ^{2}\left[\ln N, \varepsilon^{-1}\right]+N_{0}^{-2}\right\}, \quad(x, t) \in \bar{G}_{h}
$$

for the function $\omega_{U}^{[2]}(x, t)$, which is the solution of problem (B.16a), (5.11), where $v(x, t)=$ $U(x, t)$. Taking into account estimates (B.17), (B.19), we have the estimate

$$
\left|u(x, t)-z^{[2]}(x, t)\right| \leqslant M\left\{N^{-2} \min ^{2}\left[\ln N, \varepsilon^{-1}\right]+N_{0}^{-2}\right\}, \quad(x, t) \in \bar{G}_{h},
$$

and also the $\varepsilon$-uniform estimate

$$
\left|u(x, t)-z^{[2]}(x, t)\right| \leqslant M\left[N^{-2} \ln ^{2} N+N_{0}^{-2}\right], \quad(x, t) \in \bar{G}_{h} .
$$

Also, the following estimates are valid:

$$
\begin{array}{r}
\left|u(x, t)-\bar{z}^{[2]}(x, t)\right| \leqslant M\left\{N^{-2} \min ^{2}\left[\ln N, \varepsilon^{-1}\right]+N_{0}^{-2}\right\}, \quad(x, t) \in \bar{G} ; \\
\left|u(x, t)-\bar{z}^{[2]}(x, t)\right| \leqslant M\left[N^{-2} \ln ^{2} N+N_{0}^{-2}\right], \quad(x, t) \in \bar{G},
\end{array}
$$

where $\bar{z}^{[2]}(x, t),(x, t) \in \bar{G}$ is the bilinear interpolant constructed from the values of the function $z^{[2]}(x, t),(x, t) \in \bar{G}_{h}$. Estimates (B.20) and (B.21) are unimprovable with respect to the values of $N, N_{0}, \varepsilon$ and $N, N_{0}$, respectively.

\section{References}

[1] P. A. Farrell, A. F. Hegarty, J. J. H. Miller, E. O'Riordan, and G. I. Shishkin, Robust Computational Techniques for Boundary Layers, Chapman \& Hall/CRC, Boca Raton, FL, 2000.

[2] P. A. Farrell, P. W. Hemker, and G. I. Shishkin, Discrete approximations for singularly perturbed boundary value problems with parabolic layers, in three parts: J. Comput. Math., 14 (1996), No. 1, pp. 71-97; J. Comput. Math., 14 (1996), No. 2, pp. 183-194; J. Comput. Math., 14 (1996), No. 3, pp. $273-290$.

[3] P. A. Farrell, J. J. H. Miller, E. O'Riordan, and G. I. Shishkin, A uniformly convergent finite difference scheme for a singularly perturbed semilinear equation, SIAM J. Numer. Anal., 33 (1996), No. 3, pp. 11351149 . 
[4] P. W. Hemker, G. I. Shishkin, and L. P. Shishkina, The use of defect correction for the solution of parabolic singular perturbation problems, ZAMM, 77 (1997), No. 1, pp. 59-74.

[5] P. W. Hemker, G. I. Shishkin, and L. P. Shishkina, E-uniform schemes with high-order time-accuracy for parabolic singular perturbation problems, IMA J. Numer. Anal., 20 (2000), No. 1, pp. 99-121.

[6] P. W. Hemker, G. I. Shishkin, and L. P. Shishkina, High-order time-accurate schemes for parabolic singular perturbation problems with convection, Russ. J. Numer. Anal. Math. Model., 17 (2002), No. 1, pp. $1-24$.

[7] V. L. Kolmogorov and G. I. Shishkin, Numerical methods for singularly perturbed boundary-value problems modeling diffusion processes, in: Singular Perturbation Problems in Chemical Physics (J. J. H. Miller, ed.), John Willey \& Sons, New York, 1997, pp. 181-362.

[8] O. A. Ladyzhenskaya, V. A. Solonnikov, and N. N. Ural'tseva, Linear and Quasilinear Equations of Parabolic Type, Nauka, Moscow, 1967, in Russian; English transl.: Translations of Mathematical Monographs, 23, American Mathematical Society, Providence, RI, 1968.

[9] G. I. Marchuk and V. V. Shaidurov, Increasing the Accuracy of Solutions of Difference Schemes, Nauka, Moscow, 1979, in Russian.

[10] J. J. H. Miller, E. O'Riordan, and G. I. Shishkin, Fitted Numerical Methods for Singular Perturbation Problems. Error Estimates in the Maximum Norm for Linear Problems in One and Two Dimensions, World Scientific, Singapore, 1996.

[11] H.-G. Roos, M. Stynes, and L. Tobiska. Numerical Methods for Singularly Perturbed Differential Equations. Convection-Diffusion and Flow Problems, Springer-Verlag, Berlin, 1996.

[12] A. A. Samarskii, Theory of Difference Schemes, 3rd edn., Nauka, Moscow, 1989, in Russian; English transl.: The Theory of Difference Schemes, Marcel Dekker, Inc., New York, 2001.

[13] G. I. Shishkin, Grid approximation of singularly perturbed boundary-value problems with convective terms, Sov. J. Numer. Anal. Math. Modelling, 5 (1990), No. 2, pp. 137-187.

[14] G. I. Shishkin, Grid Approximations of Singularly Perturbed Elliptic and Parabolic Equations, Ural Branch of Russian Acad. Sci., Ekaterinburg, 1992, in Russian.

[15] G. I. Shishkin, Approximation of solutions of singularly perturbed boundary-value problems with a parabolic boundary layer, Zh. Vychisl. Mat. Mat. Fiz., 29 (1989), No. 7, pp. 963-977, in Russian; English transl.: USSR Comput. Math. Math. Phys., 29 (1989), No. 4, pp. 1-10.

[16] G. I. Shishkin, Grid approximations of singularly perturbed elliptic equation in domains with characteristic bounds, Sov. J. Numer. Anal. Math. Modelling., 5 (1990), No. 4\&5, pp. 327-343.

[17] G. I. Shishkin, Approximation of solutions of singularly perturbed boundary-value problems with a corner boundary layer, Zh. Vychisl. Mat. Mat. Fiz., 27 (1987), No. 9, pp. 1360-1374, in Russian;English transl.: USSR Comput. Maths. Math. Phys., 27 (1987), No. 5, pp. 54-63. 